\title{
The feasibility of prospectively studying maternal cardiovascular changes from before conception
}

\begin{abstract}
Amita A Mahendru ${ }^{1}$, Thomas R Everett ${ }^{1}$, Carmel M McEniery ${ }^{2}$, Ian B Wilkinson ${ }^{2}$ and Christoph C Lees ${ }^{1,3}$
There is compelling evidence that factors before pregnancy and around implantation may have a bearing on maternal cardiovascular adaptation to pregnancy and subsequent pregnancy outcome. Prospective studies from before pregnancy are associated with difficulties in recruitment, low conception rates, early pregnancy loss and low retention of participants during pregnancy and postpartum follow-up. The objective of this study was to establish the feasibility of recruiting to; conducting and completing a prospective cohort study from before pregnancy to the postpartum period. One-hundred and forty-three women planning to conceive were recruited. They underwent detailed cardiovascular measurements including brachial and central blood pressures, cardiac output, aortic stiffness and pulse wave reflection, metabolic function and platelet aggregation. Once pregnant, the cardiovascular assessments were repeated at intervals throughout pregnancy and postpartum. Of 143 women, 101 women conceived within 18 months. Seventy-one had viable pregnancies at 10-14 weeks. Among the 70 live-births, three women developed preeclampsia (PE) and two had intrauterine growth restriction. Two were lost to follow-up. It is feasible to recruit women who are planning to conceive, conduct prepregnancy cardiovascular assessments and follow them up during pregnancy. Based on the current data, approximately half the women recruited will have healthy ongoing pregnancies. This information would allow the design of a study, powered for pregnancy complications such as PE, to enable investigation of the 'cause and effect' relationship between abnormal cardiovascular function and pregnancy complications.

Hypertension Research (2013) 36, 698-704; doi:10.1038/hr.2013.24; published online 11 April 2013
\end{abstract}

Keywords: cardiovascular; feasibility; preeclampsia; prospective; prepregnancy

\section{INTRODUCTION}

Normal pregnancy is associated with profound maternal cardiovascular changes in order to ensure optimal conditions for fetal growth. Prospective studies from the preconception period in women with normal pregnancies have demonstrated that most of the cardiovascular changes occur just after implantation. ${ }^{1-3}$ Abnormal prepregnancy cardiovascular function ${ }^{4}$ and maternal hemodynamic maladaptation in very early pregnancy are observed particularly in women with early onset preeclampsia $(\mathrm{PE})$ and intrauterine growth restriction (IUGR) implying a possible association between events at implantation and uteroplacental insufficiency. ${ }^{5}$

Cardiovascular and metabolic dysfunction is seen in pregnancies affected by $\mathrm{PE}$ beginning from late first trimester, ${ }^{6}$ and persisting postpartum. ${ }^{7-9}$ The abnormal maternal cardiovascular function seen in PE or IUGR could either be a manifestation, or the cause, of uteroplacental insufficiency and/or may exist before pregnancy. The temporal and causative links between maternal cardiovascular and metabolic dysfunction, manifestation of uteroplacental insufficiency and cardiovascular disease later in life in pregnancies affected by PE still remain elusive. ${ }^{10}$
A prospective cohort study of cardiovascular and metabolic characteristics from prepregnancy to the postpartum period would enable investigation of the 'cause and effect' relationship between pregnancy complications and cardiovascular dysfunction, along with the timing and extent of pregnancy-induced cardiovascular changes. However, there are potential difficulties in conducting prepregnancy cohort studies. ${ }^{11}$ A large number of pregnancies is needed to investigate differences in cardiovascular adaptation in pregnancy complications such as PE, with an incidence of $3-5 \%$. There is a $25-30 \%$ probability of clinical pregnancy per menstrual cycle ${ }^{12,13}$ and one-third of women who conceive will experience a pregnancy loss. ${ }^{13,14}$ Moreover, in a recent large-scale prospective study, only one out of four women who were recruited before pregnancy were followed up. ${ }^{15}$

In order to estimate the required sample size for investigation of cardiovascular changes in pregnancy complications, it is necessary to establish the feasibility of recruitment, conception and live birth rates and compliance with follow-up investigations as per protocol during normal pregnancy beginning from before pregnancy. The objective of this study was to determine the feasibility of recruitment to and

${ }^{1}$ Fetal Medicine Department, Rosie Hospital, Addenbrooke's Hospital, Cambridge University Hospitals Foundation Trust, Cambridge, UK; ${ }^{2}$ Clinical Pharmacology Unit, University of Cambridge, Cambridge, UK and ${ }^{3}$ Biomedical Sciences, Katholieke Universiteit, Leuven, Belgium.

Correspondence: Dr CC Lees, Fetal Medicine Department, Rosie Hospital, Addenbrooke's Hospital, Cambridge University Hospitals Foundation Trust, Hills Road, Cambridge CB2 OQQ, UK.

E-mail: christoph.lees@addenbrookes.nhs.uk

Received 5 November 2012; revised 9 January 2013; accepted 14 January 2013; published online 11 April 2013 
completion of a prospective cohort study of cardiovascular function from before pregnancy to the postpartum period.

\section{METHODS}

Design

This was a prospective feasibility study of recruitment and completion of follow-up for a cohort study of maternal cardiovascular function from preconception to postpartum.

\section{Participants}

One-hundred and forty-three women planning to conceive were recruited. The inclusion criteria were: nulliparous women, previous normal pregnancies, previous PE/IUGR and previous unexplained recurrent miscarriages (RM), who were between 18-50 years of age. PE was defined by the criteria laid by the International Society for the Study of Hypertension in Pregnancy ${ }^{16}$ and IUGR was defined as birth weight below third percentile, ${ }^{17}$ in the absence of congenital infection or aneuploidy. Unexplained RM was defined as $\geqslant$ consecutive three miscarriages at $<12$ weeks, where investigations such as thrombophilia screen, cytogenetic examination of products of conception, pelvic ultrasound and parental peripheral blood karyotyping were negative and no specific problem was detected. ${ }^{18}$ The exclusion criteria were history of thrombophilia or diabetes.

\section{Recruitment}

Recruitment was via open advertisements in the hospital, general practice (GP) surgeries, preschool groups and local newspaper over a period of 11 months. Additionally, women with previous PE/IUGR were identified from the delivery unit database and fetal medicine clinic lists, those with RM were identified from the clinic lists and invited to participate via letters if they were planning to get pregnant.

\section{Study setting}

This study was conducted at a single tertiary referral center.

\section{Study protocol}

The study protocol is described in Figure 1. Women were recruited before conception and had baseline cardiovascular assessment and blood tests as described below. They then performed digital home urine ovulation tests and pregnancy tests as described in the protocol. The day of ovulation was estimated by adding one day to the day luteinizing hormone surge was detected. The day of the first positive pregnancy test using sensitive digital urine pregnancy test kits was reported as the implantation day. ${ }^{19}$ Women who became pregnant were then followed up with serial ultrasound scans and repeat cardiovascular assessments in very early pregnancy, in second and third trimester and 3-4 months postpartum. Women who did not get pregnant at

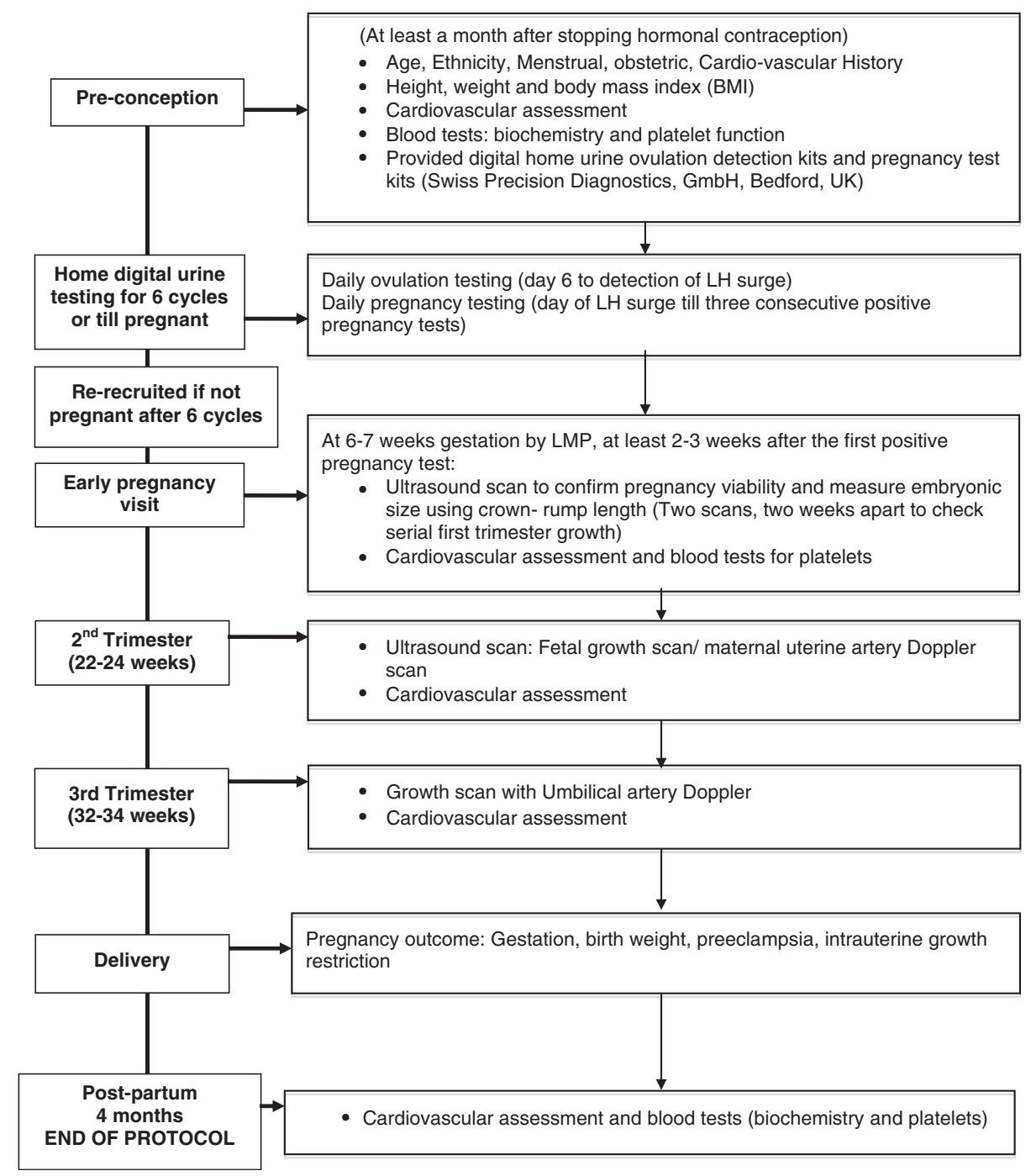

Figure 1 Study protocol. 
the end of the 6 cycles or who suffered either miscarriage or ectopic pregnancy but wanted to continue with the study were rerecruited. Their baseline cardiovascular assessments were not repeated.

\section{Cardiovascular assessment}

Cardiovascular measurements were performed after 10 min rest in the supine left lateral position. Participants were requested to abstain from caffeinated drinks for $4 \mathrm{~h}$ before the visit. Brachial blood pressure (BP) and heart rate were measured in the non-dominant arm using an automated blood pressure measuring device (Omron-M7) that has been validated for use in pregnancy, ${ }^{20}$ using standard cuff sizes according to the arm circumference of the participant. Radial artery waveforms were obtained with a high-fidelity micromanometer (SPC-301; Millar Instruments, Houston, TX, USA) from the wrist, and a corresponding central waveform was generated with a validated transfer function (SphygmoCor; AtCor Medical, Sydney, Australia). Central systolic $\mathrm{BP}$, mean arterial pressure and aortic augmentation index were determined using the integrated software. ${ }^{21,22}$ Aortic stiffness was assessed by measuring the carotid-femoral (aortic) pulse wave velocity with the same device. ${ }^{23}$ The carotid-femoral distance was measured using calipers. Cardiac output, cardiac index and stroke volume, were assessed using a non-invasive, validated inert gas rebreathing technique (Innocor, Innovision A/S, Odense S, Denmark). ${ }^{24,25}$ Peripheral vascular resistance was calculated from the formula:

Peripheral vascular resistance $\left(\right.$ dynes $\left.\mathrm{s}^{-1} \mathrm{~cm}^{-5}\right)=$ mean arterial pressure $(\mathrm{mm} \mathrm{Hg}) \times 80 /$ cardiac output $\left(1 \mathrm{~min}^{-1}\right)$.

All measurements were performed in duplicate by a single, trained investigator (AAM), and the mean values were used in subsequent analyses. The within- and between-observer measurement reproducibility values for the aortic augmentation index were in agreement with previously published data. ${ }^{26}$

\section{Blood tests}

Biochemical analysis of blood was performed prepregnancy, repeated in late first trimester along with the booking bloods and at the postpartum visit. This included markers of dyslipidaemia: total cholesterol, triglyceride, high-density lipoprotein cholesterol, low-density lipoprotein cholesterol, cholesterol:highdensity lipoprotein ratio; markers of glucose homeostasis (plasma glucose, hemoglobin Alc) and renal function: serum creatinine and estimated glomerular filteration rate. Platelet function in whole blood was analyzed prepregnancy, in very early pregnancy at 6 weeks and postpartum. Platelet function in whole blood was analyzed using Multiple Electrode impedance Aggregometry (Multiplate analyzer, Dynabyte Medical, Munich, Germany) by determining platelet aggregation on addition of adenosine diphosphate as an agonist.

\section{Ultrasound scans}

First trimester growth was assessed by measurement of embryonic crown rump length (CRL) by serial ultrasound scans at $6-7,8-9$ and fetal CRL at 10-14 weeks of gestation from last menstrual period (LMP) in all women with a regular 28-day cycle, and where this was not the case they were scheduled from the date of the luteinizing hormone surge. The embryonic CRL was measured transvaginally at 6-7 and 8-9 weeks by placing the calipers at the outer side of the crown and rump of the embryo in a longitudinal, midsagittal section. ${ }^{27}$ The fetal CRL at 10-14 weeks was imaged transabdominally in a midsagittal plane with the fetal spine longitudinally in view and the maximum crown to rump length was measured to establish the gestational age. ${ }^{28}$ Second and third trimester growth was assessed by growth charts with ultrasound measurements of fetal abdominal circumference ${ }^{28,29}$ and expected fetal weight ${ }^{30}$ at 20 weeks, 22-24 weeks and 32-34 weeks gestation.

\section{Study outcomes}

The primary outcome of the study was to provide information necessary to inform the feasibility of conducting a future study (prepregnancy recruitment, retention and protocol adherence) of sufficient power to investigate causative and temporal relationship between prepregnancy cardiovascular function, cardiovascular changes in pregnancy and development of pregnancy complications such as PE/IUGR associated with uteroplacental insufficiency.

\section{Sample size}

This was a feasibility study with no data available from any previous studies thus a formal sample size calculation was not performed. We expected to recruit 80 participants in order for at least 20 pregnancies in each group to be followed up throughout the pregnancy.

\section{Statistical analyses}

Statistical analyses were performed using the Statistical Package for social sciences (Version 18.0.0, 2009, SPSS, Chicago, IL, USA). The normality of distribution of the demographics data was examined with the KolmogorovSmirnov test. Data are expressed as median (interquartile range) and differences are compared using Kruskal-Wallis test. A $P$-value of $<0.05$ was considered significant.

\section{Ethics}

The study received Local Research Ethics Committee approval and written consent was obtained.

\section{RESULTS}

\section{Recruitment and study participation}

One-hundred and forty-three women planning to conceive were recruited in the prepregnancy period out of $184(80 \%)$ who were interested in the study and responded to the advertisements/invitations (Figure 2). Seventy nulliparous women, 35 women with previous normal pregnancies, 12 with previous PE/IUGR and 26 with previous unexplained RM were recruited. The response rate for the invitation letters in the prior risk groups was 7\% (18/250) for the PE/IUGR group and 21\% (26/125) for the RM group. Recruitment from healthy volunteers accounted for $72 \%(105 / 143)$ of the study population. The majority of women recruited consisted of nulliparous women $(49 \%)$ followed by $24 \%$ healthy multiparous women. Women with RM comprised $18 \%$ and those with PE/early IUGR comprised $9 \%$ of the cohort. The recruitment flow chart in figure 2 describes those who declined to participate in the study and those who were unsuitable. The demographic details of the study population are displayed in Table 1 . The median body mass index was $25 \mathrm{~kg} \mathrm{~m}^{-2}$ and most $(125,87 \%)$ were Caucasians reflecting our local demography. ${ }^{31}$ All except two nulliparous women, two RM women and one parous woman were nonsmokers.

\section{Conception rates and follow-up}

Ninety-one of 143 women (64\%) conceived in 18 months. There were total of one-hundred and one pregnancies in 143 women including those who conceived twice during the study period (Figure 2). The median time to conceive including the months they had been trying to conceive before participating in the research was 5 months (interquartile range 2-7 months) and this constituted a median of two cycles after entry to the study entry (interquartile range 1-5 months). The miscarriage rate was 25/101 (25\%) (Table 2). Two participants were lost to follow-up, one just after the pregnancy and another moved to another area. Therefore, the lost to follow-up rate following recruitment was $2 \%$.

\section{Protocol adherence for cardiovascular tests}

The cardiovascular assessments were performed at median 13 days of menstrual cycle in the prepregnancy period, at 6 weeks in early pregnancy, 23 weeks in the second trimester, 33 weeks in the third trimester and at 16 weeks postpartum (Table 3 ). Ninty-three percent of pregnant participants completed all the cardiovascular tests as per protocol. After the prepregnancy baseline tests, the blood biochemistry was repeated at median of 12 weeks gestation in late first trimester and at the last postpartum visit, with $90 \%$ of participants 


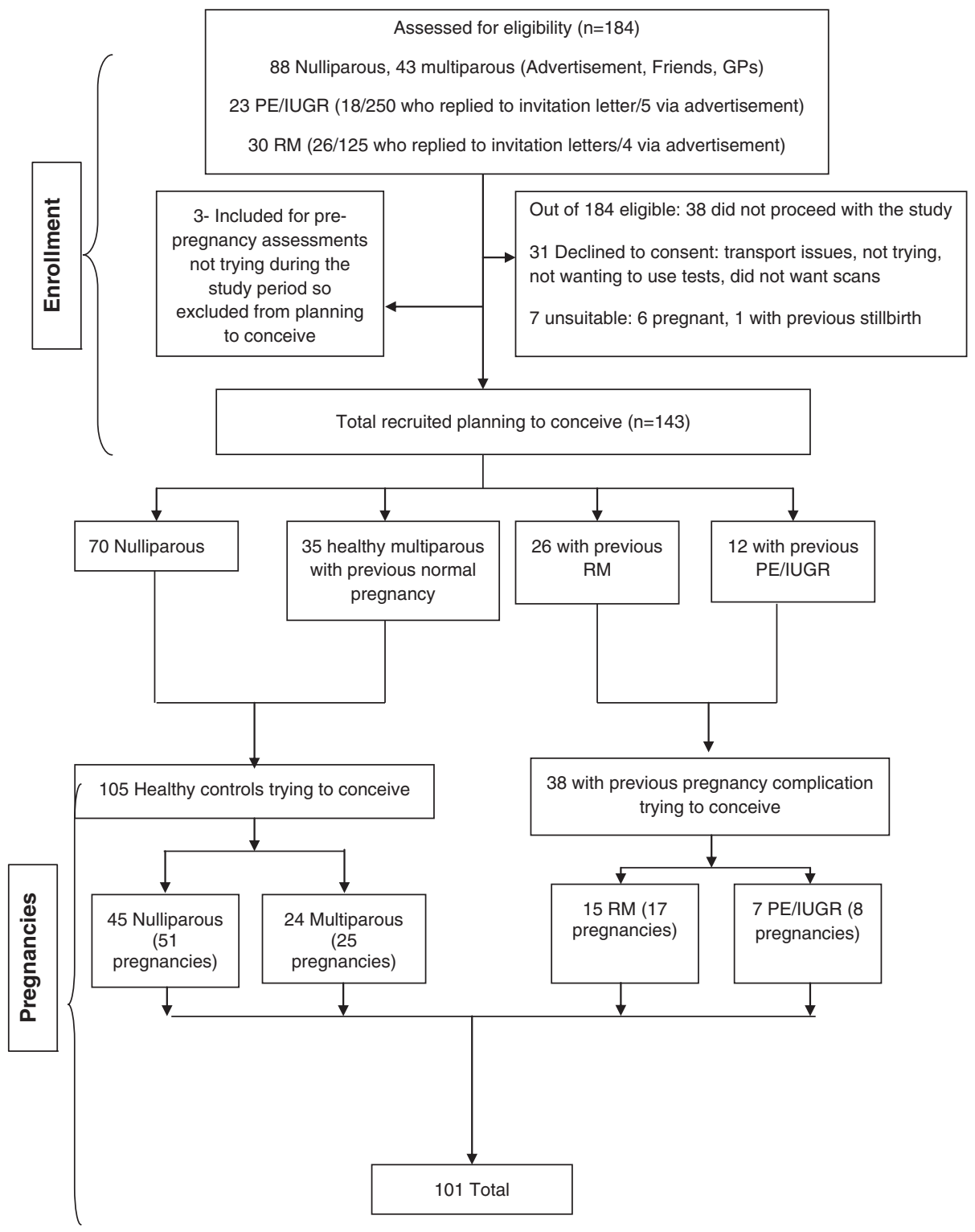

Figure 2 Recruitment flow chart describing enrollment to the study and total pregnancies.

Table 1 Demographic details of the study population

\begin{tabular}{lcccc}
\hline & \multicolumn{2}{c}{ Controls $(\mathrm{n}=105)$} & & \\
\cline { 2 - 3 } Characteristics & Nulliparous $(\mathrm{n}=70)$ & Multiparous $(\mathrm{n}=35)$ & PE/IUGR $(\mathrm{n}=12)$ & Recurrent miscarriage $(\mathrm{n}=26)$ \\
\hline Age, years & $30(28-32)^{*}$ & $35(33-38)$ & $37(32-38)$ & $36(33-41)$ \\
Caucasian & $86 \%$ & $91.4 \%$ & $80 \%$ & $88 \%$ \\
Height, meters & $1.64(1.61-1.69)$ & $1.63(1.59-1.71)$ & $1.61(1.57-1.65)$ & $71(61-86)$ \\
Weight, kg & $65(59-73)$ & $68(59-76)$ & $63(57-71)$ & $25(23-32)$ \\
BMI, $\mathrm{kgm}^{-2}$ & $24(22-28)$ & $25(22-29)$ & $25(22-29)$ & $1.67(1.62-1.72)^{*}$ \\
\hline
\end{tabular}

Abbreviations: BMI, body mass index, IUGR, intrauterine growth restriction; PE, preeclampsia.

Data are median (IQR)

${ }^{*} P<0.05$ by Kruskal-Wallis test for differences between all the four groups: Nulliparous, multiparous women with previous healthy pregnancy; women with previous PE/IUGR; women with previous recurrent miscarriage. 
Table 2 Definitions of pregnancy outcomes

\begin{tabular}{|c|c|c|}
\hline Outcome & Definition & Study outcome \\
\hline Miscarriage $<6$ weeks & $\begin{array}{l}\text { Early pregnancy loss of }<6 \text { weeks following a positive pregnancy test or no demonstrable viable pregnancy at } \\
\text { the } 6 \text { weeks scan followed by a 'missed' miscarriage. }\end{array}$ & $13 \%(13 / 101)$ \\
\hline Miscarriage $>6$ weeks & $\begin{array}{l}\text { Early pregnancy loss between } 6 \text { and } 12 \text { weeks of pregnancy after a viable pregnancy was confirmed at the } 6 \\
\text { weeks scan. }\end{array}$ & $12 \%(12 / 101)$ \\
\hline Ectopic pregnancy & Pregnancy located outside the uterus confirmed on ultrasound scan or by histological examination. & $4 \%(4 / 101)$ \\
\hline Preeclampsia ${ }^{a}$ & $\begin{array}{l}\text { Diastolic } \mathrm{BP} \geqslant 110 \mathrm{~mm} \mathrm{Hg} \text { on } \geqslant \text { one occasion, or } \geqslant 90 \mathrm{~mm} \mathrm{Hg} \text { on } \geqslant \text { two occasions at least } 4 \mathrm{~h} \text { apart, in } \\
\text { combination with significant proteinuria ( } \geqslant 300 \mathrm{mg} 24 \mathrm{~h} \text { or } 2+\text { dipstick; International Society for the Study } \\
\text { of Hypertension in Pregnancy). } 16\end{array}$ & $\begin{array}{l}\text { 4\% (2/57) (Controls) } 17 \% \\
\text { (1/6) (Previous PE/IUGR) }\end{array}$ \\
\hline $\begin{array}{l}\text { Intrauterine } \\
\text { growth restriction }\end{array}$ & Birth weight below third percentile, ${ }^{17}$ in the absence of congenital infection or aneuploidy. & $\begin{array}{l}2 \%(1 / 57) \text { (controls) } 17 \% \\
(1 / 6) \text { (previous PE/IUGR) }\end{array}$ \\
\hline
\end{tabular}

Abbreviations: BP, blood pressure; IUGR, intrauterine growth restriction; PE, preeclampsia..

The denominator for early pregnancy outcomes is 101 (total pregnancies) and that for PE and IUGR is 57 (in the normal group) and 6 in those with previous PE/IUGR.

aPE and IUGR are grouped together as uteroplacental insufficiency.

Table 3 Protocol adherences for cardiovascular assessments, blood biochemistry and platelet aggregometry in 71 viable pregnancies

\begin{tabular}{|c|c|c|c|c|}
\hline & $\begin{array}{c}\text { Timing of } \\
\text { assessmenta }\end{array}$ & $\begin{array}{l}\text { Cardiovascular } \\
\text { assessments }^{\mathrm{b}}\end{array}$ & $\begin{array}{c}\text { Blood } \\
\text { biochemistry }\end{array}$ & $\begin{array}{c}\text { Platelet } \\
\text { aggregometry }{ }^{b}\end{array}$ \\
\hline $\begin{array}{l}\text { Prepregnancy, days } \\
\text { of menstrual cycle }\end{array}$ & $13(6-21)$ & $71(100 \%)$ & 69 (97\%) & 69 (97\%) \\
\hline $\begin{array}{l}\text { Early pregnancy, } \\
\text { weeks of gestation }\end{array}$ & $6(6-7)$ & 69 (97\%) & - & $66(93 \%)$ \\
\hline $\begin{array}{l}\text { Late first trimester, } \\
\text { weeks of gestation }\end{array}$ & $12(10-13)$ & - & 63 (89\%) & - \\
\hline $\begin{array}{l}\text { Second trimester, } \\
\text { weeks of gestation }\end{array}$ & $23(23-24)$ & $68(96 \%)$ & - & - \\
\hline $\begin{array}{l}\text { Third trimester, } \\
\text { weeks of gestation }\end{array}$ & $33(32-34)$ & 67 (94\%) & - & - \\
\hline $\begin{array}{l}\text { Postpartum, weeks } \\
\text { from delivery }\end{array}$ & $16(14-17)$ & 66 (93\%) & $64(90 \%)$ & $61(86 \%)$ \\
\hline
\end{tabular}

a Data are median (interquartile range).

bata are number (percentage of total 71 viable pregnancies where data was complete).

completing all the tests as per the study protocol. The platelet aggregometry during pregnancy was repeated in early pregnancy and then postpartum and $86 \%$ of participants completed all the tests as per protocol.

\section{Pregnancy outcomes}

Two out of seventy-one women (3\%) had fetal abnormality: one had a fetus with chromosomal abnormality and underwent termination of the pregnancy at 14 weeks; the other fetus had a major cardiac anomaly and died in the neonatal period (Table 2, Figure 3). Nulliparous women and those with previous healthy pregnancies were considered together and 50/57 (88\%) controls had a normal pregnancy. Three of 57 (5\%) women had a pregnancy affected by uteroplacental insufficiency syndrome (one case of IUGR diagnosed at $<34$ weeks, two cases of PE-both diagnosed after 37 weeks, one of which had IUGR as well). All seven women with RM had normal pregnancies. Among women with previous PE/IUGR, 2/6 (33\%) had a pregnancy affected by uteroplacental insufficiency: one had PE and IUGR diagnosed at $<34$ weeks, the other had IUGR diagnosed at $>37$ weeks.

\section{DISCUSSION}

We demonstrate that it is feasible to recruit, conduct and complete a study of cardiovascular function from before pregnancy to the postpartum period. Most women conceived at a median of two cycles and used the ovulation and pregnancy test kits on a regular basis. The conception rate was similar in healthy nulliparous and multiparous women. Five percent of pregnancies were complicated by $\mathrm{PE}$ and/or IUGR consistent with the quoted incidence of PE. ${ }^{32}$ Most women (98\%) were followed up during pregnancy as per the protocol. The cardiovascular changes in normal pregnancies will be described separately in another paper.

$\mathrm{PE}$ and cardiovascular disease share similar adverse risk factors, however, it is not known if the prepregnancy risk factors predispose to both cardiovascular dysfunction and PE. ${ }^{10}$ Pregnancies complicated by $\mathrm{PE}$ are associated with cardiovascular dysfunction, ${ }^{6,33}$ however, the timing and mechanism of the cardiovascular dysfunction are not known because most studies on cardiovascular function begin from the late first trimester. ${ }^{6,34,35}$ There is paucity of longitudinal studies of cardiovascular function from before conception. ${ }^{1,2,36,37}$ This may be because of variations in retention rates, conception rates per menstrual cycle, the miscarriage rate and the 'drop-out' rates during pregnancy. ${ }^{11}$

A greater reduction in central systolic BP than in brachial BP is seen in early pregnancy ${ }^{3}$ and second trimester ${ }^{36}$ and changes in central systolic BP may reflect uteroplacental perfusion better due to the proximity of the aorta to the uteroplacental bed. ${ }^{3}$ However, there is little information about exact extent and timing of changes in central systolic BP, pulse wave velocity and pulse wave analysis between women with normal pregnancies and those with PE due to lack of longitudinal studies.

Currently, early risk prediction and diagnosis, increased surveillance and early delivery remain the management options for PE. Future research into identification of high-risk women before pregnancy and in very early pregnancy may help in improving screening for PE and may lead to development of new interventions in the prepregnancy period to prevent PE. An improved understanding of changes in the postpartum period may also enable to understand mechanisms of cardiovascular disease and prevention of cardiovascular disease by controlling postpartum risks and early treatment opportunities.

Assuming an incidence of PE of 5\%, this means that for one woman developing PE, 40 women must be recruited prepregnancy, of whom 30 will become pregnant and 20 will have viable pregnancies. 


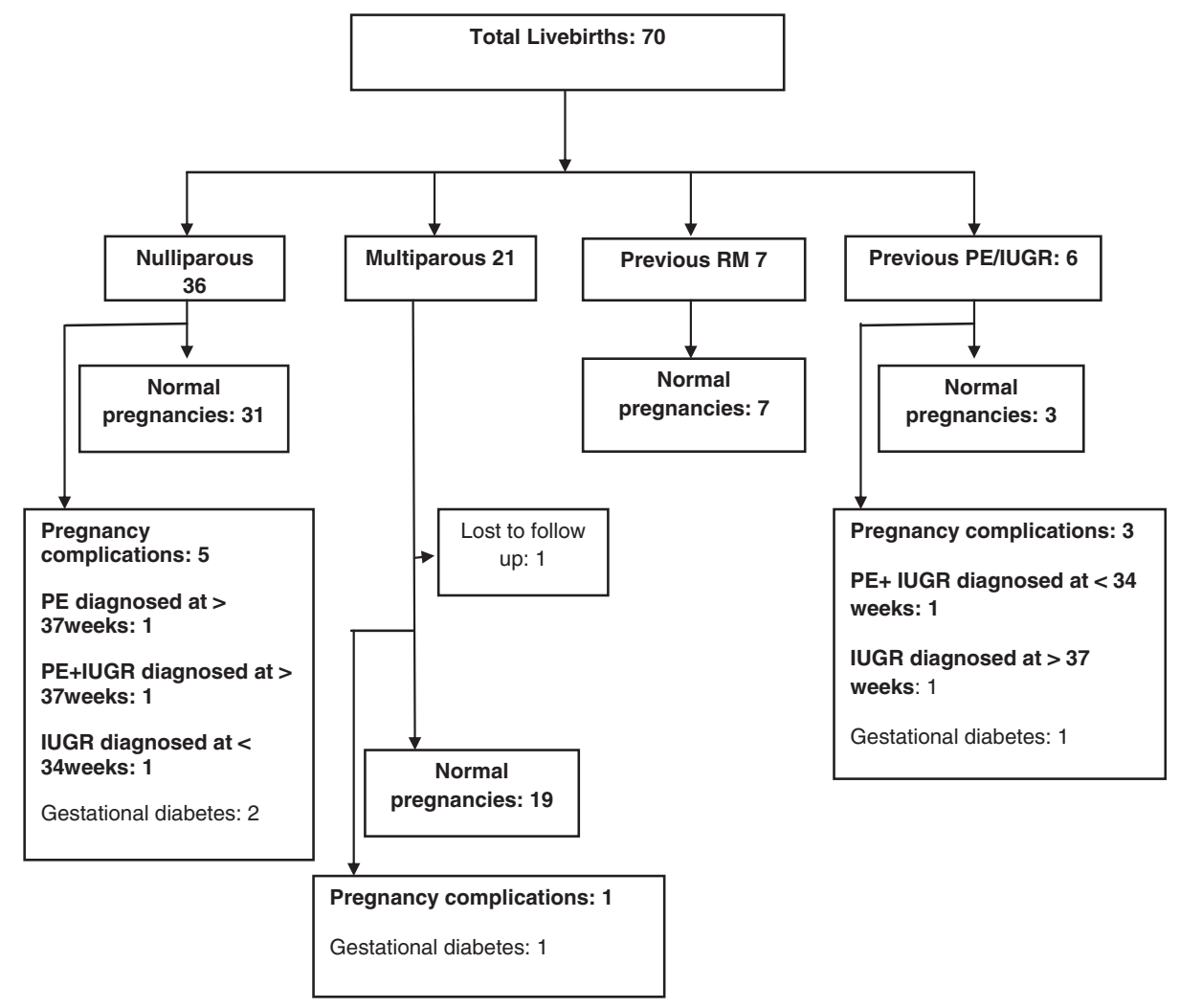

Figure 3 Pregnancy outcomes in 70 live-births.

The ratio of 40 recruited: one case of PE cardiovascular changes in a large number of healthy pregnancies need to be investigated for every one case of PE. This is necessarily resource expensive and depends on the conception, miscarriage and study retention rates.

Though it may be possible to enrich the population by recruiting high-risk individuals, women with previous PE/IUGR are known to have cardiovascular dysfunction ${ }^{38}$ and increased blood pressure, even before pregnancy. ${ }^{39}$ Their baseline prepregnancy measurements may not, therefore, be reliable to study the 'cause and effect' relationship between PE and preexisting cardiovascular risk factors. To do this would require the recruitment of nulliparous women where cardiovascular function cannot have been affected by previous pregnancy. We found that $7-14 \%$ participants did not complete the entire study protocol for cardiovascular tests or blood tests for a combination of reasons including moving away, inconvenience to attend postpartum visits, problems with equipment and transport of blood samples. A prospective study would clearly have to account for the missing data as well while calculating the sample size.

The conception rate was similar in nulliparous and multiparous women $(64 \%)$. The incidence of uteroplacental insufficiency-PE and/or IUGR is higher in women with previous PE/IUGR (33\%), consistent with their previously published recurrence rate. ${ }^{40}$ In nulliparous women, the incidence of PE and/or IUGR was $5 \%$ as expected.

An interesting and to us unexpected finding was that women with previous PE/IUGR were particularly difficult to recruit not only because of difficulties in their identification before pregnancy, but they were less likely to be planning to conceive again. Only $7 \%$ women responded to the invitation. As having had PE/IUGR would be likely to have already affected their cardiovascular function, they are a particularly important group to study.

We, therefore, demonstrate that it is possible to recruit women before pregnancy when they are trying to conceive and conduct a longitudinal study of maternal cardiovascular function in order to identify the exact extent and timing of changes at various points in pregnancy. The pregnancy follow-up and retention rate is good with a relatively low 'drop-out' rate. These data will allow estimates for a sample size for definitive future prospective cohort studies starting from before pregnancy.

\section{CONFLICT OF INTEREST}

The authors declare no conflict of interest.

\section{ACKNOWLEDGEMENTS}

We wish to thank all the women who participated in the study. The ovulation and pregnancy tests were provided free of cost by Swiss Precision Diagnostics, GmbH (SPD) Bedford. AAM was supported by Cambridge Fetal Care and Flexibility and Sustainability funding from the Cambridge University Hospital NHS Foundation Trust. TRE was funded by Evelyn Trust. IBW and CMM are both British Heart Foundation Fellows and are supported by the Cambridge Biomedical Research Centre (NIHR).

Author contributions: CCL/IBW conceived the study design. AAM wrote the study protocol, obtained ethics and performed the study. CCL and AAM were involved in writing, and IBW, CMM, TRE reviewed the final manuscript. 
1 Robson SC, Hunter S, Boys RJ, Dunlop W. Serial study of factors influencing changes in cardiac output during human pregnancy. Am J Physiol 1989; 256: H1060-H1065.

2 Capeless EL, Clapp JF. Cardiovascular changes in early phase of pregnancy. Am J Obstet Gynecol 1989; 161: 1449-1453.

3 Mahendru AA, Everett TR, Wilkinson IB, Lees CC, McEniery CM. Maternal cardiovascular changes from pre-pregnancy to very early pregnancy. J Hypertens 2012; 30: 2168-2172.

4 Magnussen EB, Vaten LJ, Lund-Nilsen TI, Salvesen KA, Smith GD, Romundstad PR. Prepregnancy cardiovascular risk factors as predictors of pre-eclampsia: population based cohort study. BMJ 2007; 335: 978-978.

5 Duvekot JJ, Cheriex EC, Pieters FA, Peeters LL. Severely impaired fetal growth is preceded by maternal hemodynamic maladaptation in very early pregnancy. Acta Obstet Gynecol Scand 1995; 74: 693-697.

6 Bosio PM, McKenna PJ, Conroy R, O'Herlihy C. Maternal central hemodynamics in hypertensive disorders of pregnancy. Obstet Gynecol 1999; 94: 978-984.

7 Yinon Y, Kingdom JC, Odutayo A, Moineddin R, Drewlo S, Lai V, Cherney DZ, Hladunewich MA. Vascular dysfunction in women with a history of preeclampsia and intrauterine growth restriction: insights into future vascular risk. Circulation 2010; 122: $1846-1853$

8 Melchiorre K, Thilaganathan B. Maternal cardiac function in preeclampsia. Curr Opin Obstet Gynecol 2011; 23: 440-447.

9 Evans CS, Gooch L, Flotta D, Lykins D, Powers RW, Landsittel D, Roberts JM, Shroff SG. Cardiovascular system during the postpartum state in women with a history of preeclampsia. Hypertension 2011; 58: 57-62.

10 Wen SW, Xie RH, Tan H, Walker MC, Smith GN, Retnakaran R. Preeclampsia and gestational diabetes mellitus: pre-conception origins? Med Hypotheses 2012; 79: 120-125.

11 Smith GCS. Periconceptual and Early Pregnancy Approach. In Pijnenborg R, Brosens I and Romero R (eds) Placental Bed Disorders (1st ednCambridge University Press, Cambridge, 2010, pp. 243-255.

12 Zinaman MJ, Clegg ED, Brown CC, O'Connor J, Selevan SG. Estimates of human fertility and pregnancy loss. Fertil Steril 1996; 65: 503-509.

13 Wilcox AJ, Weinberg CR, O'Connor JF, Baird DD, Schlatterer JP, Canfield RE, Armstrong EG, Nisula BC. Incidence of early loss of pregnancy. N Eng/ J Med 1988, 319: 189-194.

14 Mahendru A, Daemen A, Everett T, Wilkinson I, McEniery C, Abdallah Y, Timmerman D, Bourne T, Lees C. Impact of ovulation and implantation timing on first trimester crown-rump length and gestational age. Ultrasound Obstet Gynecol 2012; 40: 630-635.

15 Southampton Women's Survey. Available at http://www.mrc.soton.ac.uk/sws..

16 Davey DA, MacGillivray I. The classification and the definition of the hypertensive disorders of pregnancy. Am J Obstet Gynecol 1988; 158: 892-898.

17 Cole TJ, Williams AF, Wright CM. Revised birth centiles for weight, length and head circumference in the UK-WHO growth charts. Ann Hum Biol 2011; 38: 7-11.

18 Royal College of Obstetricians and Gynaecologists (RCOG) The Investigation and Treatment of Couples with Recurrent First-trimester and Second-trimester Miscarriage Green-top Guideline No. 17 RCOG Press, London, 2011.

19 Wilcox AJ, Baird DD, Weinberg CR. Time of implantation of the conceptus and loss of pregnancy. N Engl J Med 1999; 340: 1796-1799.

20 de Greeff A, Beg Z, Gangji Z, Dorney E, Shennan AH. Accuracy of inflationary versus deflationary oscillometry in pregnancy and preeclampsia: OMRON-MIT versus OMRONM7. Blood Press Monit 2009; 14: 37-40.

21 Nichols WW. Clinical measurement of arterial stiffness obtained from noninvasive pressure waveforms. Am J Hypertens 2005; 18: 3S-10S.
22 Nichols WW, Singh BM. Augmentation index as a measure of peripheral vascular disease state. Curr Opin Cardiol 2002; 17: 543-551.

23 Laurent S, Cockcroft J, Van Bortel L, Boutouyrie P, Giannattasio C, Hayoz D, Pannier B, Vlachopoulos C, Wilkinson I, Struijker-Boudier H. Expert consensus document on arterial stiffness: methodological issues and clinical applications. Eur Heart J 2006, 27: 2588-2605.

24 Gabrielsen A, Videbaek R, Schou M, Damgaard M, Kastrup J, Norsk P. Non-invasive measurement of cardiac output in heart failure patients using a new foreign gas rebreathing technique. Clin Sci (Lond) 2002; 102: 247-252.

25 Fontana P, Boutellier U, Toigo M. Reliability of measurements with Innocor during exercise. Int J Sports Med 2009; 30: 747-753.

26 Wilkinson IB, Fuchs SA, Jansen IM, Spratt JC, Murray GD, Cockcroft JR, Webb DJ. Reproducibility of pulse wave velocity and augmentation index measured by pulse wave analysis. J Hypertens 1998; 16: 2079-2084.

27 Pexsters A, Luts J, Van Schoubroeck D, Bottomley C, Van Calster B, Van Huffel S, Abdallah Y, D'Hooghe T, Lees C, Timmerman D, Bourne T. Clinical implications of intraand interobserver reproducibility of transvaginal sonographic measurement of gestational sac and crown-rump length at 6-9 weeks' gestation. Ultrasound Obstet Gynecol 2011; 38: 510-515.

28 Loughna P, Chitty L, Evans T, Chudleigh T. Fetal size and dating charts recommended for clinical Obstetric practice. Ultrasound 2009; 17: 161-167.

29 Chitty LS, Altman DG, Henderson A, Campbell S. Charts of fetal size: 3. Abdominal measurements. Br J Obstet Gynaecol. 1994; 101: 125-131.

30 Yudkin PL, Aboualfa M, Eyre JA, Redman CW, Wilkinson AR. New birthweight and head circumference centiles for gestational ages 24 to 42 weeks. Early Hum Dev 1987; 15: 45-52.

31 Breeze AC, Statham H, Hackett GA, Jessop FA, Lees CC. Perinatal postmortems: what is important to parents and how do they decide? Birth 2012; 39: 57-64.

32 Steegers EA, von Dadelszen P, Duvekot JJ, Pijnenborg R. Pre-eclampsia. Lancet 2010; 376: $631-644$

33 Melchiorre K, Sutherland GR, Baltabaeva A, Liberati M, Thilaganathan B. Maternal cardiac dysfunction and remodeling in women with preeclampsia at term. Hypertension 2011; 57: 85-93.

34 Fujime M, Tomimatsu T, Okaue Y, Koyama S, Kanagawa T, Taniguchi T, Kimura T. Central aortic blood pressure and augmentation index during normal pregnancy. Hypertens Res 2012; 35: 633-638.

35 Khalil A, Jauniaux E, Cooper D, Harrington K. Pulse wave analysis in normal pregnancy: a prospective longitudinal study. PLoS One 2009; 4: e6134.

36 Chapman AB, Abraham WT, Zamudio S, Coffin C, Merouani A, Young D, Johnson A, Osorio F, Goldberg C, Moore LG, Dahms T, Schrier RW. Temporal relationships between hormonal and hemodynamic changes in early human pregnancy. Kidney Int 1998; 54 2056-2063.

37 Hale S, Choate M, Schonberg A, Shapiro R, Badger G, Bernstein IM. Pulse pressure and arterial compliance prior to pregnancy and the development of complicated hypertension during pregnancy. Reprod Sci 2010; 17: 871-877.

38 Melchiorre K, Sutherland GR, Liberati M, Thilaganathan B. Preeclampsia is associated with persistent postpartum cardiovascular impairment. Hypertension 2011; 58: 709-715.

39 Mahendru AA, Everett TR, McEniery M, Wilkinson IB, Lees CC. Cardiovascular function in women with recurrent miscarriage, preeclampsia and/or intrauterine growth restriction. J Matern Fetal Neonatal Med 2012; 26: 351-356.

40 Sibai BM, Mercer B, Sarinoglu C. Severe preeclampsia in the second trimester: recurrence risk and long-term prognosis. Am J Obstet Gynecol 1991; 165 1408-1412. 